\title{
Disección de arteria vertebral en pacientes que recibieron terapia de manipulación espinal. serie de casos.
}

\section{Vertebral artery dissection in patients who had spinal manipulative therapy. case series.}

\author{
María L Espinosa ${ }^{1}$, Alejandra C Dykstra ${ }^{1}$, Héctor A Muñoz Gómez ${ }^{1}$, Raúl R Piedrabuena ${ }^{1,2}$ \\ 1 Universidad Católica de Córdoba. Facultad de Ciencias de la Salud. Clínica Universitaria Reina Fabiola Servicio de Neurología Córdoba-Argentina \\ 2 Instituto Modelo de Neurología Lennox. Correspondencia: María Laura Espinosa Servicio de Ortopedia y traumatología Clínica Universitaria Reina Fabiola. \\ Oncativo 1248-X5004FHP-Córdoba, Argentina; e-mail: marialauraespinosa1990@ gmail.com.
}

\section{Resumen}

En este trabajo se describen casos clínicos con disección de la arteria vertebral en pacientes que recibieron terapia de manipulación espinal.

\section{Introducción}

La disección de arterias cervicales (DAC) es una etiología importante de accidente cerebrovascular (ACV) en pacientes jóvenes, representando entre el $8 \%$ y el $25 \%$ de los casos, se clasifica en dos grandes grupos, aquellas espontánea y secundaria a traumatismos. Si bien la incidencia de disección de arteria carótida Interna (DACI) es más alta que la de arteria vertebral (DAV), la primera se relaciona en mayor medida con procesos infecciosos previos y dilatación aneurismática en neuroimágenes, $\mathrm{y}$ a segunda aparece en contexto de traumatismos menores dentro del mes previo al evento. Desde el punto de vista clínico la presentación con cefalea es típica en los casos de DACI, en cambio la cervicalgia y los síntomas neurológicos secundarios a isquemia cerebral son más frecuentes en la $\mathrm{DAV}^{1,2}$.

Dentro de las probables complicaciones arteriales que provoca la disección, se pueden encontrar oclusión arterial (ausencia de flujo sanguíneo); estenosis, que implica un estrechamiento de la luz; dilatación aneurismática, es decir, un agrandamiento focal de la luz arterial y el diámetro externo; hematoma mural con sangrado agudo o subagudo dentro del engrosamiento de la pared del vaso afectado. De ellas, la estenosis y oclusión arterial, son las responsables de muchos de los casos de ACV en adultos jóvenes y son características imagenológicas más frecuentes en la DAV ${ }^{1,3}$.

Las diferencias encontradas entre ambos territorios arteriales, se podría explicar en parte por su origen embrionario y características anatómicas. Las arterias de la circulación anterior son esencialmente móviles en su trayectoria cervical, protegida por músculos y tejido adiposo, pero expuesta a mayor compromiso por daño endotelial, estado protrombótico y activación de proteasas en casos infecciosos, principalmente en contexto de infección de vías aéreas. Mientras que la arteria vertebral (AV) está anclada a la columna cervical, lo que podría convertirla en más vulnerable a las manipulaciones mecánicas ${ }^{1,4}$.

La terapia de manipulación espinal (TME), ampliamente empleada para el tratamiento de dolor cervical, consiste en terapia manual donde la articulación vertebral se moviliza pasivamente entre el rango de límites anatómicos. A menudo 
implica una alta velocidad y empuje de baja amplitud, es una técnica en la que las articulaciones se ajustar rápidamente. La presencia de enfermedad osteofítica cervical u otra variación anatómica pueden predisponer o aumentar la probabilidad de lesión de la AV durante la extensión y rotación cefálica, siendo el segmento V3 el más vulnerable ${ }^{4-6}$.

Las revisiones respecto a seguridad de la TME han sido contradictorias a lo largo de los años. En 1934, se expone el primer informe médico-legal expresando la posible complicación cerebrovascular después de la misma. En 1947, se presenta el primer caso de DAC después de recibir manipulación quiropráctica ${ }^{2}$. Una revisión bibliográfica del 2017 donde se analizan los eventos adversos descritos por TME, considerando al ACV, cefalea y DAV, se encontró que cincuenta y cuatro revisiones (46\%) expresaron que TME es seguro; 15 de ellas (13\%) informan que es dañino y $49(42 \%)$ fueron neutrales o no estaba claro. Además, se concluye que en trece revisiones se expresan estimaciones de incidencia para eventos adversos graves por TME y éstos varían de 1 en 20.000 a 1 en 250.000.000 manipulaciones ${ }^{4}$. Rothwell y colegas revisaron la base de datos administrativa para identificar pacientes con ACV en territorio vertebro basilar, y determinaron que esta patología fue 5 veces más probable en quienes habían visitado un quiropráctico dentro de la semana previa al evento $(4,5 \%)$ en comparación con controles $(0,9 \%)^{7}$. Smith y asociados llevan a cabo otro estudio de diseño caso-control en menores de 60 años demostrando que de los 51 casos de DAV, 7 (14\%) recibieron TME dentro de 30 días del ACV en comparación con el 3\% de los controles, concluyendo que esta terapia aumenta 6 veces las posibilidades de complicaciones cerebrovasculares ${ }^{8}$.

Se mantiene la recomendación de sospechar DAV en pacientes que presentan síntomas neurológicos luego de TME, con el objetivo de lograr rápido diagnóstico y eventual tratamiento ya que corresponde a una de las causas más conocidas de $\mathrm{ACV}$ en paciente joven (10-25\%). En este reporte de casos, presentamos a dos pacientes con trastorno metabólico mal controlado, que presentaron ACV en territorio posterior, secundario a DAV, en contexto de tratamiento kinésico cervical.

\section{Caso clínico 1}

Paciente masculino de 52 años de edad, consulta por guardia por alteración del campo visual e hiperglucemia. Sus antecedentes personales patológicos son diabetes mellitus tipo II (mal control y poca adherencia al tratamiento), hipertensión arterial (HTA), hiperplasia prostática benigna, ex tabaquista (abandonó el hábito hace 5 años), ACV isquémico previo (año 2016).

Inicia 10 días previo a la consulta con cervicalgia, recibió tratamiento con TME, agregó síndrome vertiginoso a la semana que se autolimita y en las últimas 48 horas alteración campimétrica con visión en túnel bilateral y cefalea retroocular, asociado a hiperglucemia. Los síntomas neurológicos por los cuales consulta iniciaron 8 horas posteriores a última sesión de fisioterapia. Examen neurológico: alteración campimétrica periférica con conservación de visión macular bilateral, constatado por campimetría digital, alucinaciones visuales complejas, paresia crural izquierda $+4 / 5$ e hiperreflexia secuelar. Se sospecha ACV subagudo (NIHSS 3 y RANKIN M $2)$. Se realiza resonancia magnética $(\mathrm{RMN})$ de cerebro objetivando lesión cortico-subcortical occipital bilateral de territorio de ambas arterias cerebrales posteriores y en lóbulo cerebeloso izquierdo, todas ellas se comportan hiperintensas en T2 y FLAIR con restricción en difusión, a favor infarto agudo. Para su estudio etiológico se completa AngioRMN de cerebro: AV derecha presenta reducción de calibre en tercio proximal con presencia placa fibrocálcica, AV izquierda presenta oclusión subtotal proximal con presencia de trombo libre en tercio medio de la misma; ecocardiograma con hipertrofia del ventrículo izquierdo y FEVI $70 \%$; laboratorio con perfil lipídico alterado, hemoglobina glicosilada elevada, glucemias seriadas con malos controles a pesar de insulinización. Se inicia antiagregación dual (AAS 100mg/día + clopidogrel 75mg/día) y estatinas (rosuvastatina 40mg/día).

A las 48 horas se realiza arteriografía por sustracción digital (ASD), en la que se confirma disección oclusiva desde el origen de la AV izquierda hasta su segmento V2, con revascularización distal por intermedio de ramos musculares que llena de forma retrógrada V3; hipoplasia de AV derecha; circulación anterior de características normales. Se descarta displasia fibromuscular con angiografía de vasos renales.

Por diagnóstico de ACV en fosa posterior secundario a disección intra y extracraneal de AV izquierda, se decide anticoagulación (ACO) en conjunto con servicio de hematología: switch con acenocumarol (3mg/día VO) y enoxaparina (60mg $\mathrm{c} / 12 \mathrm{hs} \mathrm{SC})$. 
El cuarto y al sexto día de internación presenta intercurrencia neurológica (deterioro del estado de conciencia/déficit motor) se suspende ACO, se repite RMN de cerebro: en la primera ocasión se objetiva extensión del área isquémica hacia tálamo derecho y en segunda oportunidad isquemia talámica e hipocampal bilateral, temporal derecha y pedúnculos cerebelosos con sufusión
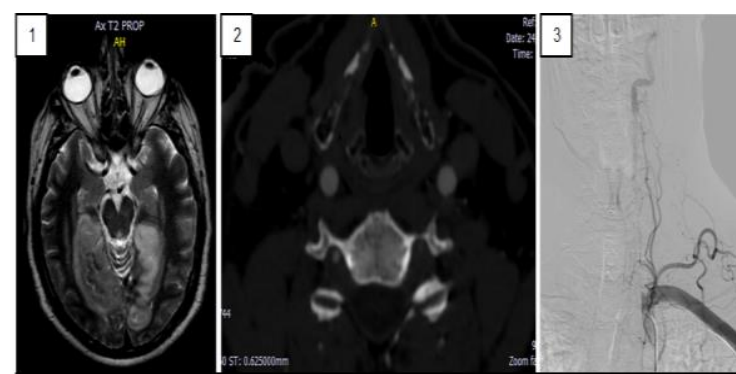

Paciente masculino de 52 años de edad. (1)RMN de cerebro: lesión hiperintensa en T2 cortico-subcortical occipital bilateral con extensión a tálamo e hipocampo bilateral. (2)AngioTC: oclusión proximal de AV Izquierda. (3)Arteriografia por sustracción digital: disección oclusiva de AV Izquierda desde su origen hasta V2.

hemorrágica en región occipital derecha. Por lo cual se restaura doble antiagregación. Se mantuvo estable neurológicamente, luego de 19 días de internación y una vez estables los parámetros metabólicos de indica alta institucional con plan de neurorehabilitación y seguimiento.

\section{Caso clínico 2}

Paciente masculino de 42 años derivado de centro oftalmológico por hemianopsia. Sus antecedentes personales patológicos son HTA, diabético mal controlado (HbA1c 10.92), hemoneumotorax a repetición, Cushing exógeno (resuelto hace 2 años), cefalea, ex-tabaquista. Inicia con la clínica hace 10 días, cefalea holocraneana de moderada intensidad asociada a contractura muscular cervical. Realizó dos sesiones de fisioterapia, comenzando con clínica neurológica luego de $3 \mathrm{hs}$ de la última sesión.

Examen neurológico: hemianopsia homónima derecha, cervicalgia, alucinaciones visuales complejas, palinopsias, sutil ataxia izquierda, parestesias en manos. NIHSS 4 mRS 2.

Se realiza RMN de cerebro objetivando lesión cortico-subcortical occipital izquierda hiperintensa en FLAIR, restricción en DWI y ADC compatible con ACV isquémico. Se realiza además angioTC de cerebro y vasos del cuello donde se objetiva ausencia del flujo de la AV derecha en su tercio inferior y flujo filiforme distal. Se instauran antihipertensivos e insulina. Ecocardiograma y de vasos normales. ASD a los 7 días del ingreso, informa estenosis severa del segmento V2 de la AV derecha, hipoplásica, probablemente secundaria a disección, con circulación anterógrada favorable. Se indica doble antiagregación (AAS $100 \mathrm{mg}$ + clopidogrel $75 \mathrm{mg}$ ) + rosuvastatina $40 \mathrm{mg}$, se agrega quetiapina por alucinaciones visuales y topiramato por cefalea diaria. Evoluciona favorablemente en controles posteriores.

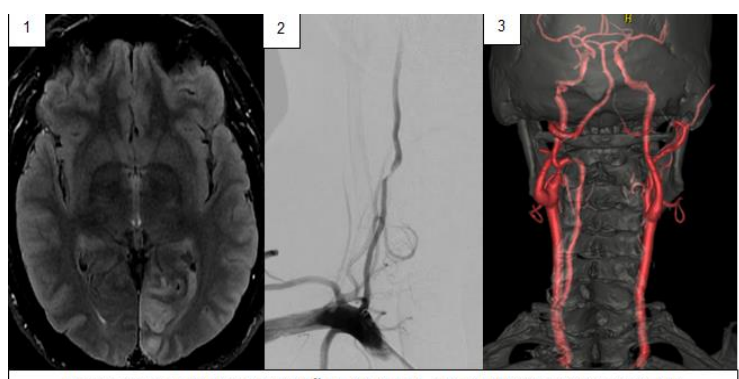

Paciente masculino de 42 años de edad. (1) RMN de cerebro: Lesión hiperintensa en Flair cortico-subcortical occipital izquierda. (2) Arteriografia por sustracción digital: estenosis severa del segmento V2 de la AV derecha, hipoplásica, secundaria a disección. (3) AngioTC: ausencia del flujo de la AV derecha en su tercio inferior y flujo filiforme distal.

\section{Discusión}

Existe controversia a la hora de considerar esta relación como causal en los pacientes cuya manifestación clínica de disección vertebral es el dolor cervical sin manifestaciones neurológicas evidentes, ya que la mayoría de los pacientes son dirigidos a terapia de manipulación cervical por este mismo síntoma; planteando el dilema en que si la disección fue causada por esta técnica o se encontraba previamente y simplemente ha sido exacerbada por la TME.

Ambos casos presentados de ACV en paciente joven secundario a disección vertebral, tienen en común la realización de fisioterapia horas previas al inicio de los síntomas neurológicos. Si bien la presentación con relación temporal de ambos hechos no siempre implica relación causal entre ellos, en ambos pacientes se descartan otras probables patologías que predisponen a la disección arterial y no se encontraron datos en su historia clínica personal ni familiar de enfermedades del tejido conjuntivo. Una característica común en estos casos es la presencia de enfermedad metabólica mal controlada, aunque no se ha encontrado una relación estrecha entre la diabetes y el riesgo de disección arterial.

\section{Conclusion}

Analizando los casos presentados, resulta mandataria la sospecha clínica de disección vertebral en pacientes que manifiestan síntomas neurológicos luego de recibir algún tipo de terapia de manipulación espinal, la rapidez en el diagnóstico permitirá brindar un tratamiento

Revista Methodo: Investigación Aplicada a las Ciencias Biológicas. Universidad Católica de Córdoba. Jacinto Ríos 571 Bo Gral. Paz. X5004FXS. Córdoba. Argentina. Tel.: (54) 3514517299 / Correo: methodo@ucc.edu.ar / Web: methodo.ucc.edu.ar |ARTICULO ORIGINAL Rev. Methodo 2019;4(2):44-48 
precoz, que se traduce en mejor pronóstico y menor discapacidad.

$\mathrm{Si}$ bien la relación entre ambas entidades se desconoce con exactitud y la evidencia científica es insuficiente para establecer la afirmación de que la TME puede ser causa de disección arterial, los informes clínicos sugieren que las fuerzas mecánicas sobre la columna vertebral juegan un papel importante en un número considerable de DAC y en la mayoría de los estudios controlados de la población han encontrado una asociación causal en pacientes jóvenes, aunque su incidencia sea baja y la causalidad difícil de probar.

Recomendamos considerar la posibilidad de DAC e informar a los pacientes, aunque resulta controvertido considerar segura o no ésta técnica; se han reportado a lo largo de los años una cantidad importante de casos, como nuestros pacientes, en los cuales hubo una estrecha relación entre TME y el inicio de la clínica neurológica.

\section{Summary}

This paper describes clinical cases with dissection of the vertebral artery in patients who received spinal manipulation therapy

\section{Introduction}

The spinal manipulative therapy (SMT) used for cervical pain, includes all procedures where the hands or mechanical devices are used to mobilize, adjust, manipulate, apply traction, massage, stimulate or otherwise influence the spine and paraspinal tissues with the aim of influencing the patient's health 1,4. Nowadays many studies correlate SMT with serious adverse events, such as cervical artery dissection (CAD) and stroke due to it 3. CAD is a tear or haematoma in the wall of the neck arteries and is recognised as an important cause of ischaemic stroke in young to middle aged people, accounting for $2 \%$ of all ischaemic stroke but $10-25 \%$ in those under 55 years of age. The aetiology may be spontaneous or traumatic but is thought to involve an underlying intrinsic susceptibility coupled with exposure to an external trigger, such as minor trauma or SMT, 2.

\section{Clinical cases}

In this case series two male patients are included, 52 and 42 years old, hypertensive, poor controlled diabetics, overweighted patients. The first arrived with vertigo, bilateral tunnel vision, 48 hours cervical and retroocular pain. The second arrived with homonymous hemianopia, visual hallucinations and left retroocular pain. Both had had SMT 12 hours before. MRIs were performed: T2 y FLAIR hyperintensities and DWI restriction suggested posterior territory ischemia. Angio RMN: subtotal left vertebral artery occlusion and free-floating thrombus in its middle third. The angio-CT showed absence of flow of the right vertebral artery in its lower third and filiform flow in its upper third. Both had stroke suggestive findings that were probably secondary to vertebral artery dissection. They were given double antiplatelet (aspirin 100mg/day and clopidogrel $75 \mathrm{mg} /$ day), rosuvastatin $40 \mathrm{mg} /$ day. The arteriography of the patients confirmed the vertebral artery dissection, they did not meet endovascular treatment criteria. The first patient had dissection and occlusion from the beginning to its V2 segment, the second had severe stenosis of the V2 segment of the vertebral artery. In the first case we decided to treat with acenocoumarol (3 $\mathrm{mg}$ /day orally) y enoxaparina (60 mg/12hs SC), he had two neurological events and the stroke extended. At the fifth day of treatment the patient's occipital lesions bled and we went back to double antiplatelet treatment.

\section{Conclusion}

We analyzed the cases and results mandatory the quick clinical suspect of vertebral artery dissection in patients with neurological symptoms after SMT, the diagnosis will allow us to give a right treatment at the right time, which means a better prognosis and less inability. We recommend to consider the possibility of CAD and inform our patients about the association between CAD and SMT, even though the scientific evidence is not clear and its controversial to consider this technic safe or not. There have been several cases reported through the years, as our patients who were closely related the causality between SMT and the beging of the neurological symptoms.

\section{Bibliografía}

1. Debette S 1, Grond-Ginsbach C, Bodenant M, Kloss M, Engelter S, et al "Differential features of carotid and vertebral artery dissections: the CADISP study". Neurology. 2011 Sep 20;77(12):1174-81. doi: 10.1212/WNL.0b013e31822f03fc. Epub 2011 Sep 7.

2. Zhu Zhu, Xu Y., Wang Y., Zhou Z. Han X., el al. Chinese Cervicocephalic artery dissection study (CCADS): rationale and protocol for a multicenter prospective cohort study. Zhu et al. BMC Neurology (2018) 18:6. DOI 10.1186/s12883-018-1011-x. 
3. Thomas LC. Cervical arterial dissection: An overview and implications for manipulative therapy practice. Man Ther. 2016 Feb; 21:2-9. doi: 10.1016/j.math.2015.07.008. Epub 2015 Jul 29.

4. Nielsen SM1, Tarp S1, Christensen R1, Bliddal H2, Klokker L1, Henriksen M The risk associated with spinal manipulation: an overview of reviews. Syst Rev. 2017 Mar 24;6(1):64. doi: 10.1186/s13643-017-0458-y.

5. Ernst E. Manipulation of the cervical spine: a systematic review of case reports of serious adverse events, 1995-2001. Med J Aust. 2002 Apr 15;176(8):376-80.

6. WHO Guidelines on Basic Training and Safety in Chiropractic.: WHO Press, World Health Organization; 20 Avenue Appia, 1211 Geneva 27, Switzerland

7. Rothwell PM1, Norris JW. Cerebrovascular complications of therapeutic neck manipulation. The need for reliable data on risks and risk factors. J Neurol. 2002 Aug;249(8):1105-6.

8. Biller J., Sacco R.L., Albuquerque F.C., Demaerschalk B.M, Fayad, P, et al. Cervical Arterial Dissections and Association With Cervical Manipulative Therapy: A Statement for Healthcare Professionals From the American Heart Association/American Stroke Association. Originally published1 Oct 2014. DOI: $\quad$ 10.1161/STR.0000000000000016. Stroke. 2014;45:3155-3174.

\section{Palabras claves}

DISECCIÓN ARTERIA VERTEBRAL, ACCIDENTE CEREBROVASCULAR, FISIOTERAPIA, TERAPIA DE MANIPULACIÓN ESPINAL

\section{Keywords}

VERTEBRAL ARTERY DISSECTION, STROKE, PHYSIOLOGICAL THERAPY, SPINAL MANIPULATIVE THERAPY.

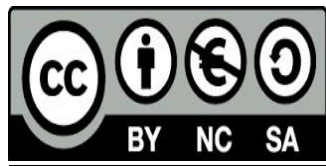

